Gdańsk 2018, Nr. 38

\title{
Sonja Klimek
}

Departement für Sprachen und Literaturen der Universität Fribourg, Schweiz

/ Oxford Centre for Life-Writing, Wolfson College, University of Oxford https://doi.org/10.26881/sgg.2018.38.02

\section{„So fällt die Feder mir vor Wehmuth aus der Hand“ - Verstummen und Nicht-mehr-Schreiben-Können als Topos im Klagegedicht der Frühaufklärung}

\begin{abstract}
Trauer gilt seit der Antike als sprachlos machende Emotion. Dennoch gibt es in wohl allen Literaturen aller Zeiten Klagelyrik. Wie aber kann jemand, der eigentlich vor Trauer verstummt, überzeugend als ,Sprecher' eines Klagegedichts vorgestellt werden? Anhand zweier Beispiele aus dem Barock wird gezeigt, mit welchen Topoi dieses Dilemma im Gedicht selbst thematisiert und somit zur eigentlichen ,inventio' des Gedichtes gemacht wurde. Abschließend wird am Beispiel dreier Dichterinnen der Frühaufklärung untersucht, wie Frauen gegen das als ,weiblich` konnotierte Verstummen vor Trauer anschrieben.
\end{abstract}

Schlüsselwörter: Klagelyrik, Frühaufklärung, Unsagbarkeitstopos, Trauer, Frauenbildung

"The quill drops from my woefull hand" - Falling silent and losing the capacity to write as a topos in lamentation poetry from the Early Enlightenment. Since antiquity, grief has been regarded as an emotion that makes us fall silent. Nevertheless, all times and cultures have produced lamentation poetry. But how can someone who is in grieving be convincingly presented as the 'speaker' of a poem? Referring to two Baroque poems, this paper shows which topoi authors have used to make this dilemma the 'inventio' of a poem. Finally, three examples of early Enlightenment women poets are studied who, in their lamentation poetry, fight the silencing which is assumed to be typically 'female'.

Keywords: lamentation poetry, Early Enlightenment, topos of the inexpressible, learned women

\section{Trauer als sprachlose Emotion. Poetologische Debatten} um ihre Darstellbarkeit

Der Frage nach der Darstellung von Gefühlen in Kunstwerken kommt seit der Antike eine besondere Bedeutung zu. Neben starken Emotionen wie Liebe, Eifersucht und Wut spielt in den poetologischen Debatten dabei gerade die Trauer eine zentrale Rolle, wird sie doch seit jeher als eine von sich aus, sprachlose ' Gefühlslage angesehen. ${ }^{1}$ Menschen, die soeben die Nachricht vom Tod eines geliebten Menschen empfangen haben, halten keine Ansprachen, vielmehr äußert sich ihre akute Trauer meist mimisch, gestisch und lautlich-unartikuliert, etwa durch lautes Wehklagen,

1 Vgl. Claudia Benthien, Barockes Schweigen. Rhetorik und Performativität des Sprachlosen im 17. Jahrhundert, München 2006, S. 284. 
Schluchzen und Tränen, Händeringen und Haareraufen, ${ }^{2}$ oder auch in einem gänzlichen Verstummen. In der Literatur werden das Trauern und das Schweigen denn auch bereits seit Sappho als "Zwillingstopoi“3 angesehen. Und noch Joachim Heinrich Campes „Wörterbuch der Deutschen Sprach “"kennt 1810 das Adjektiv „trauerstumm“ ${ }^{4}$ Sebastian Treyz sieht hierin die „eigentümliche Ambivalenz“ oder „Paradoxie“ aller Totenklageliteratur, „,scheint doch [...] keine andere Emotion eine tiefere Beziehung zur Sprachohnmacht zu unterhalten als die angesichts der Vergänglichkeit des Lebens oder der Abwesenheit des vertrauten Anderen erlittene Trauer." Das je individuell ausgeprägte emotionale Erleben von Trauer scheint - auch heute noch - begrifflich kaum einholbar und auch für nahestehende Personen nur begrenzt miterlebbar zu sein. ${ }^{6}$

Auf der anderen Seite gehören Klagelieder und -gedichte aber zum Kernbestand vermutlich aller Literaturen der Welt. Bereits im Alten Testament wird das Halten einer rituellen Totenklage (das Singen feierlicher „Lamentationes“ oder „Threnoi“ im jüdischen Versmaß der Totenklage, so genannter, Qina') an mehreren Stellen erwähnt (z.B. Amos 8,10), und das Fehlen einer solchen wird als harte Strafe angesehen (vgl. etwa Psalmen 78,64; Jeremia 16.4f.).

Lyrisches Sprechen in Zeiten der Trauer, literarische Totenklage steht immer in einem „Spannungsfeld von Emotionalität und Kognition, von körperlicher Sprachlosigkeit und

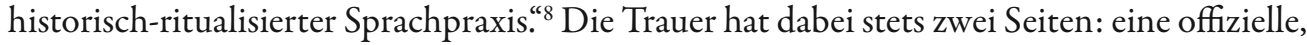
denn durch den Tod einer Person werden gesellschaftliche Strukturen verschoben, Güter wechseln ihren Eigentümer, Positionen im sozialen Gefüge werden frei und müssen ggf. neu besetzt werden. Als Phase eines Übergangs wird die Trauer daher in wohl allen Kulturen rituell geregelt. Trauer hat aber auch eine private Seite. Wenn der verstorbene Mensch geliebt wurde, so hinterlässt sein Tod eine innere Leere bei den Hinterbliebenen, die sowohl ihren sich nun veränderten Alltag als auch ihr emotionales Erleben betrifft. Diese beiden Seiten der Trauer, der für das gesellschaftliche Zusammenleben relevante Aspekt und der das Individuum verändernde, geraten dabei nicht selten in Konflikt miteinander. Trauer beim Tod naher Verwandter und Freunde zu empfinden wird etwa in der Frühaufklärung naturrechtlich als etwas ,Natürliches', dem Menschen Gemäßes angesehen. ${ }^{9}$ Andererseits gibt es aber

Vgl. Gisela Ecker, Trauer zeigen. Inszenierungen und die Sorge um den Anderen, in: dies. (Hrsg.), Trauer tragen - Trauer zeigen. Inszenierungen der Geschlechter, München 1999, S. 9-25, hier: S. 17.

Kathy Zarnegin, Einleitung, in: dies. (Hrsg.), buchstäblich traurig, Basel 2004, S. 7-15, hier: S. 7.

4 Lemma „Trauerstumm: [...] sprachlos vor Trauer, vor Schmerz“, in: Wörterbuch der Deutschen Sprache, Veranstaltet u. hrsg. v. Joachim Heinrich Campe, Vierter Theil: S und T, Braunschweig 1810, S. 866.

5 Sebastian Treyz, „Schämt euch der Wehmuth nicht, die feucht im Auge schimmert..." Tränen- und Trauerdispositive im Theater der Empfindsamkeit, in: Seraina Plotke, Alexander Ziem (Hrsg.), Sprache der Trauer. Verbalisierungen einer Emotion in historischer Perspektive, Heidelberg 2014, S. 245-281, hier: S. 245.

6 Vgl. Monika Müller, Matthias Schnegg, Unwiederbringlich. Von der Krise und dem Sinn der Trauer, Göttingen 2016, S. 23.

Vgl. Georg Hentschel, Totenklage [Artikel], in: Manfred Görg, Bernhard Lang (Hrsg.), Neues Bibellexikon, Düsseldorf 2001, S. 908-910.

8 Seraina Plotke, Alexander Ziem, Sprache der Trauer im interdisziplinären Kontext. Einführende Bemerkungen, in: dies. (Hrsg.), Sprache der Trauer. Verbalisierungen einer Emotion in historischer Perspektive, Heidelberg 2014, S. 1-17, hier S. 3.

9 Vgl. Johann Heinrich Zedler, Grosses vollständiges Universal-Lexicon Aller Wissenschaften und Künste [...], Bd. 45, 1745, 2., vollständiger photomechanischer Nachdruck durch die Akademische Druck- u. Verlags- 
oft auch klare gesellschaftliche Erwartungen an die Trauernden, ab wann sie ihre Aufgaben für das Gemeinwesen nach dem Trauerfall - zumindest schrittweise - wieder aufnehmen sollen. Dazu gehörte für zeugungsfähige Personen, sich nach Ablauf der Trauerzeit (z.B. des so genannten, Trauerjahres') wieder neu zu vermählen, um zum Fortbestand der Gemeinschaft beizutragen. ${ }^{10}$ Es gab - gerade in der Frühen Neuzeit - gesellschaftliche Vorgaben, wie Literatur dazu beitragen könne, die ,natürlichen' Gefühle der Trauer zu kanalisieren, sie gezielt auszuleben und dadurch letztlich kathartisch abzuführen.

Um solch sympathetische Regungen bei den Leser/innen zu erwecken, muss die im Gedicht dargestellte Trauer ,authentisch wirken. Bereits Horaz schrieb in seiner „Epistola ad Pisones“: „[...] si vis me flere, dolendum est / Primum ipsi tibi [...]“ (auf Deutsch: „Willst du, daß ich weine, so traure erst einmal selbst" ${ }^{\text {") }}{ }^{11}$ Allerdings richteten sich diese Worte bei Horaz zunächst einmal an den Schauspieler, der auf der Bühne eine Figur in Trauer mimen soll und dabei nicht unfreiwillig komisch wirken darf. Statt dem , authentischen 'Ausdruck eines ,genuine[n], spontane[n] Gefühl[s]" ging es bei Horaz also vielmehr um eine gewisse Selbstaffektation des Künstlers zum Zweck der überzeugenderen schauspielerischen Leistung während des Vortrags. ${ }^{12}$

Auch Nicolas Boileau nimmt diesen Gedanken 1674 in der französischen Klassik noch fast wörtlich in sein Lehrgedicht „L'art poétique " auf und bezieht ihn auf Schauspieler, die einen Unglücklichen darstellen sollen: „Il faut dans la douleur que vous vous abaissiés. / Pour me tirer des pleurs il faut que vous pleuriés."13

Im Zuge der Klassizismus-Rezeption wird diese Position in den 1730er Jahren in Deutschland u.a. von Johann Christoph Gottsched (1700-1766) prominent vertreten, der wie Boileau und später Batteux dem aristotelischen Diktum anhängt, poetische Dichtung sei immer Nachahmung. Im vierten Kapitel seines „Versuch[s] einer Critischen Dichtkunst“ beschreibt

anstalt, Graz 1997, Sp. 138-146, Lemma „Trauer-Klage, oder Todten-Klage“, hier Sp. 138: „Es ist den Menschen von Natur eingepflanzet, die Todten, und insonderheit die Angehörigen zu betrauren und zu beklagen““

10 Vgl. etwa den offenen Brief zur Frage, „O Wahrhaffte Liebe zwischen Ehe-Leuten / sich nothwendig in anderer Gesellschafft kund geben müsse?", in dem Christian Thomasius seinen erst vor wenigen Monaten verwitweten Schwager Johann von Besser ermahnte, seine unmäßige Trauer nicht durch Klagegedichte an die Öffentlichkeit zu tragen, sondern sich möglichst rasch neu zu verheiraten und so, anstatt der vergangenen Liebe hinterherzutrauern, sich an einer neuen zu erfreuen (in: ders., Allerhand bißher publicirte Kleine Teutsche Schriften / Mit Fleiß colligiret und zusammen getragen; Nebst etlichen Beylagen und einer Vorrede, Halle 1701, S. 321-340).

11 Quintus Horatius Flaccus, Ars Poetica. Die Dichtkunst. Lateinisch / Deutsch, Übers. u.m. einem Nachwort hrsg. v. Eckart Schäfer, Stuttgart 1972/2011, S. 11. Vgl. auch ebd.: „Steht die Sprache des Sprechers nicht in Einklang mit seiner Lage, wird sich unter römischen Rittern und Fußvolk Gelächter erheben.“ - Die Forderung nach der „Entsprechung von Sprache und Emotion, Charakter und Gegenstand“ geht schon auf Aristoteles Poetik zurück (vgl. Eckart Schäfer, Anmerkungen, in: Quintus Horatius Flaccus, Ars Poetica. Die Dichtkunst. Lateinisch / Deutsch. Übers. u.m. einem Nachwort hrsg. v. Eckart Schäfer, Stuttgart 1972/2011, S. 37-54, hier vgl. S. 41). - Zu ähnlichen Aussagen bei Quintilian und Cicero, vgl. Jürgen Stenzel, ,Si vis me flere... - ,Musa iocosa mea'. Zwei poetologische Argumente in der deutschen Diskussion des 17. und 18. Jahrhunderts, in: „Deutsche Vierteljahresschrift“ 48 (1974), S. 650-671, hier: S. 652-653.

12 Vgl. Jürgen Stenzel, ,Si vis me flere..., S. 652.

13 L'art poétique (1674), chant III, vers 141f. (Zitiert aus: Art poétique de Boileau-Despréaux. Nouvelle Édition, Paris 1871, S. 33.) 
Gottsched 1730 das ,poetische Rollenspiel', in dem „der Poet selbst die Person eines andern spielet" und Reden erfindet, die sich für eine bestimmte Figur in einer bestimmten Situation „schicken“ würden, ohne dass sich der Dichter selbst in der Lage dieser Figur befindet. ${ }^{14}$ Insofern ist für Gottsched also keine echte Gefühlsbeteiligung des Dichters gefragt, sondern vor allem Erfahrungswissen (wie drücken sich Menschen in emotionalen Extremsituationen aus?), „empirische Psychologie ${ }^{“ 15}$ statt eigener Emotionalität: „Man muß hier die innersten Schlupfwinkel des Herzens ausstudieret und durch eine genaue Beobachtung der Natur den Unterschied des gekünstelten, von dem ungezwungenen angemerket haben. ${ }^{\text {"16 }}$ Bei Gottsched geht es jedoch nicht mehr nur um den Schauspieler, sondern um den Poeten verschiedener Gattungen. Dies merkt man daran, dass Gottsched ausführlich einen gewissen Sonderfall behandelt, wenn nämlich Dichter ausnahmsweise auch einmal eigene und nicht nur an anderen Menschen studierte Gefühle nachahmen. Er diskutiert diese Ausnahme anhand der zu seiner Zeit viel gelesenen Klagegedichte der beiden brandenburgpreußischen Dichter Friedrich Rudolph Ludwig von Canitz (1654-1699) und Johann von Besser (1654-1729). Doch auch in diesen ,privaten Klagegedichten sieht Gottsched nur poetische Nachahmung der zwar selbst erlebten, aber im Moment des Schreibens zweifellos bereits erkalteten Gefühle, also letztlich auch nur poetische Produktion aufgrund von Beobachtung (diesmal von Selbstbeobachtung):

[ ] so viel ist gewiß, daß ein Dichter zum wenigsten dann, wann er die Verse macht, die volle Stärke der Leidenschaft nicht empfinden kann. Diese würde ihm nicht Zeit lassen, eine Zeile aufzusetzen, sondern ihn nöthigen, alle seine Gedanken auf die Größe seines Verlusts und Unglücks zu richten. Der Affect muß schon ziemlich gestillet seyn, wenn man die Feder zur Hand nehmen, und alle seine Klagen in einem ordentlichen Zusammenhange vorstellen will. ${ }^{17}$

Dieses Diktum, das in einem gewissen Widerspruch zum Horaz'schen ,si vis me flere' zu stehen scheint, wird in den 1740er Jahren Anlass eines großen Literaturstreites. Bereits Johann Jacob Bodmer empfiehlt 1741 in seinen „Critischen Betrachtungen über die poetischen Gemählde der Dichter“" „dass man niemahls schreibe, als wenn man einen Affect empfindet, und wenn man nichts mehr empfindet, die Feder niederlegt. ${ }^{\text {"18 }}$ Allerdings meint er mit „einen Affect empfinde[n]" nicht das akute persönliche Betroffensein: Bodmer glaubt mitnichten, dass man beispielsweise Klagegedichte nur verfassen dürfe, während man selbst gerade einen Todesfall zu beklagen hat, dass also die dargestellten Gefühle im eigenen Leben ihren Anlass haben müssten. Der Dichter dürfe lediglich nicht ,kalten Sinnes' schreiben (was für Gottsched geradezu die Voraussetzung „ordentliche[r]“ "Gedichte war), da sich die überzeugend echte „Sprache des Hertzens" vielmehr nur dann einstelle, wenn der Dichter sich mit Hilfe seiner „Einbildungskraft“ die traurige Situation eines Betroffenen intensiv,

14 Johann Christoph Gottsched, Versuch einer Critischen Dichtkunst. Erster Allgemeiner Theil, Joachim Birke, Brigitte Birke (Hrsg.), Berlin, New York 1973, S. 197-198.

15 Thomas Anz, Tod, Angst und Trauer in der Lyrik zwischen Barock und Aufklärung, in: „Der Deutschunterricht" 54 (2002), H. 1, S. 25-35, hier: S. 34.

16 Johann Christoph Gottsched, Versuch, S. 198.

17 Ebd., S. 198-199.

18 Johann Jacob Bodmer, Critische Betrachtungen über die Poetischen Gemählde der Dichter, Zürich 1741, S. 340 . 
wenn sie gleich abwesend und durch die Zeit und den Ort weit von uns entfernet sind, auf eine so lebhafte Weise vor das Gesicht stell[t $]$, daß das Gemüthe dadurch auf verschiedene Art in Bewegung kömmt, und eben dergleichen Leidenschaften in sich aufglimmen fühlet, als die Dinge in ihrer Anwesenheit, da sie in die Sinnen fallen, zu erregen pflegen. ${ }^{19}$

Auf diese Weise kan ein Mensch, der eine reiche Einbildungskraft in einer zarten und biegsamen Seele besitzt, Affecte annehmen, wie und wann es ihm gefällt [ ]. [ ] muntern Köpfen von einer feuerreichen Einbildungskraft wird es nicht schwer fallen, sich auf besagte Weise zu erhitzen, und einen gewissen Affect an sich zu nehmen; und alsdann dörfen [!] sie sich nur der Führung desselben überlassen, und das schreiben, was derselbe ihnen in die Gedancken giebt. Ich bin ihnen gut davor, daß mit dem Affecte zugleich auch die Figuren, als die Form seiner Reden, sich einstellen werden [...]. ${ }^{20}$

Es ist also auch laut Bodmer noch erlaubt, ja durchaus vorgesehen, auch über nicht selbst Erlebtes zu schreiben, aber nur, wenn man - als sowohl begabter wie gebildeter und gut trainierter Dichter - durch die eigene „Einbildungskraft“ fähig ist, bei sich selbst dieser Situation entsprechende Gefühlszustände hervorzurufen. Wenig später empört sich jedoch Georg Friedrich Meier, der Schüler Baumgartens, 1747 in seiner „Beurtheilung der Gottschedischen Dichtkunst" gegen jegliches Darstellen von Gefühlen, mit denen der Dichter nicht durch wahrhaftig an sich gemachte Erfahrungen vertraut ist:

Der Herr Professor [Gottsched] sagt, [...] man macht z.E. ein verliebtes, trauriges, lustiges Gedichte im namen eines andern; ob man gleich selbst weder verliebt, noch traurig, noch lustig ist. Dieses ist ohne Zweifel unmöglich. Wer eine Leidenschaft nicht selbst empfindet, der kan sie nicht nachahmen [...]. ${ }^{21}$

Hatte die Debatte in der europäischen Horaz-Rezeption u.a. bei Boileau bisher darauf fokussiert, wie der Dichter sprachlich die Wirkung der Betroffenheit erzeugen konnte, so verschob sich im frühen 18. Jahrhundert der Fokus von den Mitteln, mit denen man „Aufrichtigkeitseffekte" ${ }^{\text {“2 } 2}$ in Texten erzielen konnte, zur Person des Verfassers dieser Texte selbst. Statt nach den Techniken einer überzeugenden Darstellung fingierter Affekte fragte man vielmehr:

Wieweit muss der Dichter, der Affekte darstellt, diese selbst empfinden, empfunden haben oder in sich künstlich stimulieren; wie groß muss der Abstand zu den eigenen Affekten sein, um überhaupt dichten zu können; ist die ,Sprache der Leidenschaften', für die ja ein festes Inventar rhetorischer Figuren bereitsteht (Interjektionen, Ellipsen, Exklamationen), erlernbar; oder wieweit lässt sie sich intuitiv, ohne den Umweg über rhetorische Gelehrsamkeit sprechen ${ }^{23}$

19 Ebd., S. 341

20 Ebd., S. 343

21 Georg Friedrich Meier, Beurtheilung der Gottschedischen Dichtkunst, Halle 1747, S. 98. - Ganz ähnlich sieht es dann auch Klopstock 1759 in seinen Gedanken über die Natur der Poesie: „Von dem Poeten hier [d.h. bei der Darstellung von Trauer] nichts als Nachahmung fodern, heißt ihn in einen Akteur verwandeln, der sich vergebens als einen Akteur anstellt. Und vollends der, der seinen eigenen Schmerz beschreibt! der ahmt also sich selbst nach?" (in: Friedrich Gottlieb Klopstock, Ausgewählte Werke, hrsg. v. Karl August Schleiden, München 1962, S. 993).

22 Vgl.: Simon Bunke, Katerina Mihaylova (Hrsg.), Aufrichtigkeitseffekte. Signale, soziale Interaktionen und Medien im Zeitalter der Aufklärung, Freiburg i.Br. 2016.

23 Thomas Anz, Tod, Angst und Trauer, S. 34. 
So wurde in der ersten Hälfte des 18. Jahrhunderts das Klagegedicht zum „Muster- und zugleich Problemfall jeder ,Affekt'-Poesie “, ${ }^{24}$ weil sich hier die Frage nach der Darstellbarkeit von einer derart starken, an sich eigentlich sprachlos machenden emotionalen Regung wie der Trauer kristallisierte.

\section{Der , performative Widerspruch' ,guter' Klagelyrik und seine selbstreferentielle Thematisierung im Barock}

Jemand, der nicht trauert, kann per se keine, guten' Klagegedichte verfassen. Aber wie kann jemand, der von Gefühlen überwältigt ist, die ihn schier sprachlos machen, überhaupt einen poetischen Text produzieren, der dann auch noch höchsten formalen Ansprüchen genügen soll? Elisabeth Reber nennt dieses Dilemma den „performativen Widerspruch“ der Klagelyrik:

Der Dichter versichert, aufgrund seiner großen Trauer nicht in der Lage zu sein zu dichten und folglich auch keine Artikulation für die Trauer zu finden, wenngleich er im selben Atemzug die Emotion und ihre Auswirkungen in kunstvollen Versen beschreibt. ${ }^{25}$

In der zweiten Hälfte des 18. Jahrhunderts werden die Dichter des ,Sturm und Drang für sich die Antwort finden, dass die künstliche Form, da sich das Genie keine Fesseln anlegen lassen könne, aufgebrochen werden müsse. Wie jedoch lösten frühere Generationen für sich das Problem?

Eine gerne gewählte Möglichkeit, die „Paradoxie“, ein poetisch angemessenes und gleichzeitig überzeugendes Klagegedicht zu verfassen, bestand in der Frühen Neuzeit darin, die Unmöglichkeit des Unterfangens im Gedicht selbst zu thematisieren. Besonders eindrücklich zeigt dies ein Beispiel aus einer Gedenkschrift für den Humanisten Buxtorf aus dem Jahr 1665, das Reber zitiert:

Men'ergo lachrymas numeris constringere Metri?

Atque sub angusto limite versiculi?

Men'Spondaeorum trutina librare dolorem?

Dactyli et exacto pondere flere pedis?

Moestitiae hocce foret male contradicere nostrae:

Plangere velle foret, Plangere nolle foret,

Excedunt lachrymae nostrae numerumque modumque:

Exundant, diffluunt, mensuramque ignorant;

Et ripas spernunt quantitatum Syllabarum.

Nam Magnum flent Buxtorfium. Illustre Nomen. ${ }^{26}$

24 Dietmar Till, Transformationen der Rhetorik. Untersuchungen zum Wandel der Rhetoriktheorie im 17. und 18. Jahrhundert, Tübingen 2004, S. 422.

25 Elisabeth Reber, Gefühle nach Maß. Untersuchungen zur Trauerartikulation in den neulateinischen Epicedien für Johannes Buxtorf den Jüngeren (1599-1664), in: Seraina Plotke, Alexander Ziem (Hrsg.), Sprache der Trauer. Verbalisierungen einer Emotion in historischer Perspektive, Heidelberg 2014, S. 207-244, hier: S. 208.

${ }^{26}$ „Soll ich also meine Tränen in das Versmaß zwingen und in die enge Grenze des Versleins? Muss ich den Schmerz nach dem Maß der Spondeen abwägen? Weinen nach dem genauen Gewicht des daktylischen Fußes? Das würde bedeuten, unserer Trauer schlecht zu entsprechen: Es hieße, klagen zu wollen und gleichzeitig nicht klagen zu wollen. Unsere Tränen überschreiten den Vers und das Maß: Sie strömen über, fließen hervor und wollen nichts von einem Abzählen wissen, und sie missachten die Ufer der festgelegten Silbenquantitäten, 
Die Faktur des Gedichtes, aber auch die Schreibsituation wird in allen Arten von Casualcarmina des Barock immer wieder thematisiert, etwa in Gestalt der topischen „fliegenden Feder“, die sich in der Binnenlogik der Schreibsituation durch die aus dem Schreibanlass folgende gebotene Eile der Abfassung erklärt und gleichzeitig die „wesentliche Unvollkommenheit“ des Gelegenheitsgedichtes erklärt. ${ }^{27}$ So wird das Dilemma zur eigentlichen ,inventio des Klagegedichtes gemacht: „In aller Breite und Ausführlichkeit wird die Unfähigkeit des Autors dargelegt, vor lauter Schmerz" und unter Zeitdruck (denn die Beerdigung naht) „etwas aufzuschreiben [...]. ${ }^{\alpha 28}$ Ein späteres Beispiel findet sich in der Gedichtsammlung des dänischen Hof-Historiographen Christoph Heinrich Amthor (1677-1721). Sein Klagegedicht auf den Tod einer nahen Anverwandten beginnt folgendermaßen:

\section{Letzte Freundschaffts-Pflicht Bey dem Grabe Frauen} Susannen Jönsen/ Gebohrner Amthorin. 1709.

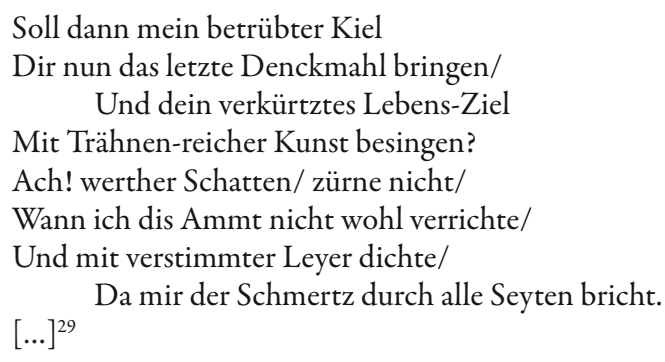

Sowohl die „Leyer“ als sympathetisches Instrument des mythischen Sängers mit ihren vor Traurigkeit „verstimmte[n] " „Seyten“ kommt vor wie auch der nicht weniger mitfühlende „Kiel“ des Schreibenden. Der Adressant dieses Gedichtes inszeniert sich also gleichzeitig in der Rolle als quasi-archaischen ,Sängers' als auch in der des modernen ,Schriftstellers', der mit der Schreibfeder in der Hand ein Manuskript als Druckvorlage für einen Separatdruck oder einen Gedichtband herstellt. Das Gedicht wird als „diejenigen Gedanken [inszeniert], die der Autor unmittelbar im Anschluss an die Todesmeldung entwickelt und niederschreibt. ${ }^{\text {“30 }}$ Der tatsächliche Schreibprozess mit seiner vermutlich langwierigen,

denn sie beweinen den großen Buxtorf, einen berühmten Namen““ (Übersetzung ebenfalls bei Elisabeth Reber, Gefühle nach Maß, S. 207).

27 Wulf Segebrecht, Das Gelegenheitsgedicht. Ein Beitrag zur Geschichte und Poetik der deutschen Lyrik, Stuttgart 1977, S. 167. - Zur „Unvollkommenheit“ der in ,Poetischen Wäldern' (als ,unbearbeitetes Rohholz') gesammelten Gelegenheitsgedichte, vgl. grundsätzlich Wolfgang Adam, Poetische und Kritische Wälder. Untersuchungen zu Geschichte und Formen des Schreibens ,bei Gelegenheit', Heidelberg 1988.

28 Wulf Segebrecht, Das Gelegenheitsgedicht, S. 167.

$29 \mathrm{C}$ [hristoph] H[einrich] Amthors, Poetischer Versuch Einiger Teutscher Gedichte und Übersetzungen: So wie er sie, theils in frembdem Nahmen, theils vor sich selber entworffen. Nebst einem Vor-Bericht, Worin zugleich die wieder seine Gedichte, und andere Staats-Schrifften, von einigen Ungenannten bisher ausgegossene Schmähungen bescheidentlich abgelehnet werden, Flensburg 1717, S. 209-213, hier: S. 209-210.

30 Wulf Segebrecht, Das Gelegenheitsgedicht, S. 171. 
entwerfenden, um- und neuschreibenden Arbeitsweise tritt hinter die Fiktion zurück, es, spreche' ein ,Ich` die Worte des Gedichts spontan aus.

\section{Die unbeschreibliche Trauer einer Mutter. Susanna Margaretha von Kuntschs Umdeutung des Timanthes-Stoffs}

Während das Gedicht auf Buxtorfs Tod noch relativ innovativ mit dem Topos von der formensprengenden Trauer umgeht, steht Amthors Text bereits an einem Übergang der Tradition. In der Frühen Neuzeit war das „Schweigen als faktischer Redeabbruch“, im Unterschied zu späteren Texten der Empfindsamkeit und des Sturm und Drang etwa, lange noch völlig „unüblich“, so Claudia Benthien. ${ }^{31}$ Stattdessen wurde die „elaborierte, syntaktisch abgerundete, dem Versmaß gemäße und von zahlreichen Stilfiguren gezierte Rede auch im höchsten

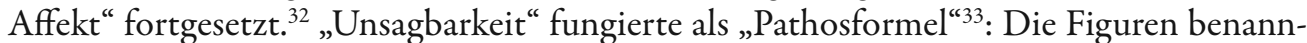
ten ihren Affekt oft einfach, anstatt ihn zu beschreiben. ${ }^{34}$ Dabei wurde auch häufig auf den antiken Topos der ,Darstellbarkeit des Undarstellbaren' am Beispiel des griechischen Malers Timanthes von Kythnos rekurriert,

der die Opferung der Iphigenie als Motiv gewählt hatte. Der Legende nach stellt der Maler die intensiven Emotionen in den Gesichtern der umstehenden Personen dar, erkennt dann aber, dass ihm die Mittel versagen, auch die Trauer des Vaters Agamemnon abzubilden. Denn neben dem Schmerz über den Verlust der Tochter hatte er sich dem Mythos zufolge auch die Schuld an ihrem Tod anzurechnen, da er selbst es war, der sie auf der Fahrt nach Troja opferte, um den von Artemis gesandten Gegenwind zu stoppen. Timantes setzt bei der Darstellung Agamemnons daher einen Kunstgriff ein: Er verhüllt das Gesicht des Vaters mit einem Schleier. Das Sichtbare wird so nicht abbildhaft, sondern als ein verweisen- des Zeichen verstanden, als , uneigentliche Darstellung des eigentlich Undarstellbaren'. ${ }^{35}$

Ein Beispiel für den kreativen Umgang mit dem Timanthes-Stoff und dem Mythos von der Opferung Iphigenies findet sich im postum veröffentlichten Gedichtband „FR[au] Margarethen Susannen von Kuntsch Sämmtliche Geist- und weltliche Gedichte“. Margaretha Susanna von Kuntsch (1651-1716) war die Tochter eines Altenburgischen Hofbeamten und hatte selbst wieder einen Hofrat geheiratet. ${ }^{36}$ In ihrer Jugend strebte sie nach höherer Bildung, u.a. nach Latein- und Französischkenntnissen, doch wurden ihr diese Studien von den Eltern

31 Claudia Benthien, Barockes Schweigen, S. 278.

32 Ebd.

33 „Pathosformeln“ sind (nach Aby Warburg, Dürer und die italienische Antike, in: Die Erneuerung der heidnischen Antike. Kulturwissenschaftliche Beiträge zur Geschichte der europäischen Renaissance, Nachdruck der Ausgabe von 1932, hrsg. v. Horst Bredekamp, Michael Diers, Berlin 1998, S. 443-450. Dargestellt bei Claudia Benthien, Barockes Schweigen, S. 290-295) epochenübergreifende „Ausdrucksform[en] menschlicher Leidenschaften [...], welche sich in affektiven Leitgebärden manifestieren“ (Claudia Benthien, Barockes Schweigen, S. 32).

34 Vgl. Claudia Benthien, Barockes Schweigen, S. 32.

35 Ebd., S. 284-285, unter Verwendung eines Zitates aus Ralf Konersmann, Der Schleier des Timanthes. Perspektiven der historischen Semantik, Frankfurt a.M. 1994, S. 13.

36 Vgl. Barbara Becker-Cantarino, Der lange Weg zur Mündigkeit. Frau und Literatur (1500 - 1800), Stuttgart 1987, S. 259. 
bald aus Anstandsgründen versagt. Herausgegeben wurde ihr Gedichtband erst 1720, vier Jahre nach ihrem Tod, und zwar von ihrem Enkelsohn Christoph Gottlieb Stockmann. ${ }^{37}$

Das Besondere an Kuntschs Sammlung sind die in der Abteilung „Düstrer Cypressen-Wald Der Hertzlich Traurenden Und Mitleidenden Sylvien" enthaltenen Klagegedichte beim Tod ihrer Kinder. Kuntsch musste im Laufe ihrer Ehejahre 12 ihrer insgesamt 13 Kinder sowie mehrere der Enkel von der ihr einzig verbliebenen Tochter früh zu Grabe tragen. Die Gedichte beim Tod ihrer Kinder sind jedoch keine Epicedien, also keine Trostgedichte. Es sind tatsächlich Klagegedichte, ein seltener Beleg für das Leid, das Eltern in ihrem Familienalltag häufig erfuhren, in dieser Zeit, in der gut die Hälfte der Kinder vor dem Erreichen des 10. Geburtstages starb. Nach einem Gedicht auf den Tod des „sechst gebohrne[n] Töchterlein[s]“" steht in der postum veröffentlichten Gedichtsammlung von 1720

\title{
Als gleiches Unglück mit dem fünftgebornen Söhnlein/ dem kleinen Chrisander, oder C. K. den 22. November 1686. durch GOttes Verhängniß sich begab.
}

\author{
Alß dort Timantes Agamemnons Schmertz/ \\ Da Iphigenien man opfern wolte/ \\ Und wie sein Vater Hertz \\ sich drob gequälet / bilden solte/ \\ Da zog er einen Flohr \\ Desselben Antlitz vor. \\ Und zeigte damit an/ \\ Es könne seinem Pinsel nicht gelingen/ \\ Wie kläglich er gethan/ \\ Recht lebhafft durch die Farben rauß zu bringen. \\ Warum? der Hertzens=Stoß \\ Sey gar zu starck und groß. \\ Was ist ein einzig mahl/ \\ Man stelle Agamemnon mich entgegen/ \\ Mich/ der des Würgers Stahl \\ Das neunte Kind hat müssen nun erlegen/ \\ Indem worauf mit Lust \\ Ich hofft/ ins Grab gemust. \\ Zwung ein so tapfrer Held/ \\ Ein König der gewohnet zu regieren/ \\ Der dmahls wolt ins Feld \\ Ein Kriegs=Heer gegen seine Feinde führen/ \\ Durch sein sonst tapfres Hertz \\ Nicht einen solchen Schmerz? \\ Ja traut der Künstler sich
}

37 Vgl. Gisela Brinker-Gabler, Deutsche Dichterinnen vom 16. Jahrhundert bis zur Gegenwart. Gedichte und Lebensläufe [1978], Frankfurt a.M. ${ }^{2} 1979$, S. 101. 
Nicht zu/ da er sonst künstlich war in schilden/

Durch seinen Pinsels Strich

Der Eltern Schmerz bey Kinder Tod zu bilden/

Daß er vielmehr verdeckt/

Was ihre Seel erschreckt/

Wer giebet mir den Muth/

Wer will mir meine Feder künstlich schärffen/

Wie jetzo wall't mein Blut/

Auf dieses Blatt mit Worten zu entwerfen/

Die ich ein Weib nur bin/

Ach! hier erstarrt mein Sinn.

Die Hand erzittert mir/

Die Feder will mir ihren Dienst versagen/

Es schüttert das Papier/

Und kann die Schmerzens=Worte nicht ertragen/

Drumm zeuge stummes Leyd

Von meiner Traurigkeit! $!^{38}$

Dieses Verstummen am Ende des Gedichtes ist mehr als jene „rhetorische (Meta-)Figur der Aposiopese ", ${ }^{39}$ bei der die Rede bewusst abbricht, ehe das eigentlich Wichtige ausgesprochen wurde. Gaby Pailer macht zu Recht darauf aufmerksam, dass hier die mit Timanthes assoziierte Frage nach der Darstellbarkeit elterlicher Trauer beim Tod eines Kindes ,von Bild auf Schrift und von der väterlichen auf die mütterliche Perspektive" verschoben wird. ${ }^{40}$ Das Gedicht folgt einem strengen rhetorischen Aufbau: Zwei mal folgen auf zwei Strophen These („Ekphrase der antiken Szene “ ${ }^{\text {«1 }}$ des Agamemnon und Beschreibung der Darstellungsproblematik für den antiken Maler Timanthes) jeweils zwei Strophen Antithese (Darstellung der eigenen Situation der Adressantin, in der der „Moment des Kindesverlusts als [...] Erlebnis des persönlichen Erstarrens und Verstummens" erfahren wird), ${ }_{4}^{42}$ gefolgt von einer Conclusio in der siebten Strophe, das Versagen sowohl der Schreibhand (als pars pro toto für den Körper der Dichterin) als auch der Feder (des Werkzeugs) als auch des Papiers (des Mediums) beim Versuch, die eigenen Gefühle zu schildern. Das Gedicht endet mit der Aufrufung des zur Klimax gesteigerten Unsagbarkeitstopos: Wenn schon Timanthes als begnadeter Maler vor dem Leid des Vaters (der noch dazu an seiner Trauer selbst schuld war) nur noch den ,andeutende[n], verhüllende[n] Darstellungsmodus “43 ${ }^{\star 3 a ̈ h l e n ~ k o n n t e, ~ w i e ~ s o l l t e ~ d a n n ~ e r s t ~ e i n e ~ u n g e l e h r t e ~}$

38 Anna Carrdus (Hrsg.), Das ,weiblich Werck' in der Residenzstadt Altenburg (1672-1720). Gedichte und Briefe von Margaretha Susanna von Kuntsch und Frauen aus ihrem Umkreis, Hildesheim, Zürich, New York 2004, S. 125-126.

39. Claudia Benthien, Barockes Schweigen, S. 283.

40 Vgl. Gaby Pailer, Poetin und Gegenstand der Poesie. Adressantenmarkierung bei Margaretha Susanna von Kuntsch, in: Claudia Hillebrandt, Sonja Klimek, Ralph Müller, Rüdiger Zymner (Hrsg.), Grundfragen der Lyrikologie, Bd. 1: Lyrisches Ich, Textsubjekt, Sprecher? Berlin, Boston [voraussichtlich 2019, im Druck].

41 Gaby Pailer, Poetin und Gegenstand der Poesie [im Druck].

42 Vgl. Gaby Pailer, Poetin und Gegenstand der Poesie [im Druck].

43 Claudia Benthien, Barockes Schweigen, S. 286. 
Frau Worte für ihre eigene Trauer als unschuldige und hilflose Mutter finden? Das „stumme Leyd“ unzähliger Mütter der Frühen Neuzeit - denn außer bei Kuntsch hat es tatsächlich, soweit mir bekannt, kaum Spuren in der Literaturgeschichte hinterlassen - wird hier, in den 1680er Jahren, wenn auch zunächst nur in nicht veröffentlichten Gedichten, als das das bereits nicht mehr Darstellbare noch Übersteigende umschrieben.

\section{Gegen das ,weibliche' Verstummen. Klagelyrik von Christiana Mariana von Ziegler und Mariane Elisabeth von Bressler}

Kuntschs Gedichtband zeigt einen um die Wende vom 17. zum 18. Jahrhundert langsam bemerkbaren gesellschaftlichen Wandel im deutschsprachigen Raum an: Neben anderen Faktoren hatte die steigende Alphabetisierungsquote dazu geführt, dass um 1700 Lesen „zur wichtigsten Freizeitbeschäftigung für Frauen des gehobenen Bürgertums s" ${ }^{\text {“4 }}$ sowie für einige adlige Damen geworden war. ${ }^{45}$ Zwar war die Frau der höheren Mittelschicht und häufig auch in der Oberschicht in ihrem Bildungsstreben noch immer abhängig von Männern, „weil es am guten Willen von Vätern, Brüdern und Ehemännern lag, ob ihr Bildung und Bücher zugänglich gemacht wurden “ ${ }^{46}$ Ausnahmen bildeten jedoch, zumindest in beschränktem Umfang, finanziell abgesicherte Witwen, die selbst über ihr Geld und ihre Zeit disponieren konnten. ${ }^{47} \mathrm{Zu}$ diesen Ausnahmen zählte die Leipziger Witwe Christiana Mariana von Ziegler, geb. Romanus, verwitwete von Könitz, spätere von Steinwehr (1695-1760). In den 1720er und 1730er Jahren führte sie in Leipzig einen der ersten philosophisch-literarischmusikalischen Salons Deutschlands, in dem neben Johann Christoph Gottsched auch Johann Sebastian Bach ${ }^{48}$ und zahlreiche aufstrebende wie etablierte Künstler und Gelehrte der Messeund Universitätsstadt verkehrten.

Diese sozialen Veränderungen führten um 1700 auch im deutschen Sprachraum zu einer Rezeption der "Querelle des Femmes“, also dem seit der Antike, vermehrt aber seit der Renaissance immer wieder aufflammenden „Streit um die Frage, ob Frauen den Männern unterlegene Mängelwesen“ oder ob sie „ebenso wie Männer mit Vernunft und Tugend begabt seien““99 Vor allem in der Frühaufklärung wurden - verbreitet durch so genannte „Moralische Wochenschriften“ - ein neues Frauenbild und Vorschläge zur „Verbesserung der weiblichen Erziehung

44 Helga Brandes, Lemma „Frau“, in: Werner Schneiders (Hrsg.), Lexikon der Aufklärung. Deutschland und Europa, München 1995, S. 126-128, hier: S. 127.

45 Vgl. Cornelia Caroline Köhler, Frauengelehrsamkeit im Leipzig der Frühaufklärung. Möglichkeiten und Grenzen am Fallbeispiel des Schmähschriftenprozesses im Zusammenhang mit der Dichterkrönung Christiana Mariana von Zieglers, Leipzig 2007, S. 13.

46 Steffen Martus, Aufklärung. Das deutsche 18. Jahrhundert - ein Epochenbild [2015], Berlin 22015, S. 392.

47 Barbara Stollberg-Rilinger, Die Aufklärung. Europa im 18. Jahrhundert, Stuttgart 2000/2011, S. 156-157.

48 Er vertonte 1725, kurz nach seiner Ankunft als Kantor an der Thomaskirche, neun ihrer KantatenGedichte (BWV 103, 108, 87, 128, 183, 74, 68, 175 und 176).

49 Kerstin Merkel, Heide Wunder, Einleitung, in: dies. (Hrsg.), Deutsche Frauen der Frühen Neuzeit. Dichterinnen, Malerinnen, Mäzeninnen, Darmstadt 2000, S. 7-17, hier S. 7. - Vgl. auch Volker Meid, Die deutsche Literatur im Zeitalter des Barock. Vom Späthumanismus zur Frühaufklärung (1570-1740), München 2009 , S. 55. 
und Bildung" an die Öffentlichkeit gebracht..$^{50}$ Zudem sahen sich die deutschen Gelehrten durch die steigende Internationalisierung des Buchmarktes und des sich beschleunigenden Ideentransfers zunehmend in Konkurrenz zum Ausland, besonders zum englisch-, französischund italienischsprachigen. Insofern ging es den Mitgliedern der diversen deutschen Sprachgesellschaften in der ersten Hälfte des 18. Jahrhunderts, wenn sie Frauen förderten, zumeist auch oder sogar vorrangig um die Behauptung ihrer eigenen kulturellen Gleichwertigkeit "in der res publica literaria“. 51

Die Zieglerin trat erstmals 1725/26, unter dem Pseudonym „de Rose“, durch Beiträge zu Johann Christoph Gottscheds Wochenschrift „Vernünftige Tadlerinnen“ an die Öffentlichkeit. In ihren Texten trat sie nachdrücklicher für das Recht der Frauen ein, an den gebildeten Debatten ihrer Zeit schreibend und publizierend teilzunehmen. 1728 erschien ihr erster Gedichtband, „Versuch in gebundener Schreib-Art“, bei Johann Friedrich Braun in Leipzig. Anders als die Kuntsch publizierte die Zieglerin ihre Gedichte also durchaus bereits zu Lebzeiten, und sie trat dabei hinter keinen männlichen Herausgeber oder Vorredner zurück. Anders als die Kuntsch präsentierte die Zieglerin in ihrem äußerst galanten Gedichtband aber fast keine durch Gedichttitel als private Totenklagen gekennzeichneten Texte. Die einzige Ausnahme bildet das Gedicht „Auf das Absterben Der Fr[au] von Breßler". 52

Es handelt sich bei dieser auch mit eigenen Briefgedichten im Band vertretenen Verstorbenen um Mariane Elisabeth von Bressler (1690-1728), ${ }^{53}$ die Frau eines Breslauer Ratsherren und Gelehrten. Wie die Zieglerin, so hatte auch die Bresslerin sich pseudonym an Gottscheds Wochenschrift beteiligt und für das Recht der schreibenden Frau plädiert.

In Zieglers Gedichtband „Versuch in gebundener Schreib-Art" sind insgesamt drei poetische Sendschreiben (d.h. Briefe in Gedichtform) an die Breslauer Dichterin von der Zieglerin in Leipzig sowie die darauf reagierenden „Antwort-Schreiben“ der Breßlerin abgedruckt, so dass ein Dialog zwischen zwei entfernt lebenden, aber sich über ihr jeweiliges Schreiben austauschenden Dichterinnen, die sich wechselseitig "Schwester" und „Freundin“ nennen, inszeniert wird. Hauptthema ist die gegenseitige Ermutigung der beiden Autorinnen, trotz des äußeren Drucks auf,gelehrte Frauenzimmer' und auch unter belastenden familiären Umständen weiterhin zu dichten und zu publizieren. In ihrem zweiten Sendschreiben versucht die Zieglerin, ihre Dichterfreundin, die vor lauter „Harm und Gram“ über den Tod

50 Vgl. Helga Brandes, Lemma „Frau“, S. 127.

51 Kerstin Merkel, Heide Wunder, Einleitung, S. 7. - Vgl. auch Anke Detken, Gekrönte Poetinnen. Gelegenheitsdichtung von Ziegler und Zäunemann, in: Sylvia Heudecker, Dirk Niefanger, Jörg Wesche (Hrsg.), Kulturelle Orientierung um 1700. Traditionen, Programme, konzeptionelle Vielfalt. Tübingen 2004, S. 263-281, hier: S. 266.

52 Christiana Mariana von Ziegler, Versuch in gebundener Schreib-Art, Leipzig 1728, S. $22-25$.

53 Nähere Informationen zu ihr findet man in den Anmerkungen zu: Barthold Heinrich Brockes, Irdisches Vergnügen in Gott. Erster und zweiter Teil. hrsg. v. Jürgen Rathje, Göttingen 2013, S. 1021. - Zwar sind nur wenige Gedichte der Bresslerin erhalten, doch spricht für eine gewisse Berühmtheit unter Zeitgenossen, dass Benjamin Neukirch ihr ein Gedicht mit dem Titel „An die schlesische Melpomene“ widmete und drei ihrer eigenen Gedichte in seiner Neukirch'schen Sammlung abdruckte. Vgl. Mirosława Czarnecka, Marianne von Bressler (1690-1728) - eine unbekannte Dichterin aus Breslau, in: Klaus Garber (Hrsg.), Stadt und Literatur im deutschen Sprachraum der Frühen Neuzeit, Bd. 2, Tübingen 1987, S. 961-972. 
ihres geliebten Ehemannes und die Erkrankung ihres Kindes nicht mehr dichten könne, trotz der traurigen persönlichen Lebensumstände zu neuer lyrischer Produktion zu motivieren:

Der Schlag ist alzu hart, klagst du, der mich betroffen

Erst meiner Augen-Lust, jetzt mein geliebtes Kind,

Bey letztern kann ich zwar noch auf genesen hoffen,

Wiewohl mir diß noch nicht die Wunden glantz verbind.

Diß ziehet meine Hand vom Säyten-Spiel zurücke,

[...] Ich bin ein kranck und Jammer-volles Weib. ${ }^{54}$

Hier wird die Trauer als ein dem Dichten entgegenstehender Gemütszustand vorgestellt. Die Zieglerin empfiehlt jedoch, nun wieder mit eigener Stimme sprechend, nachdrücklich die Abkehr vom eigenen schweren Schicksal und stattdessen die Hinwendung zum positiven Denken und zur Tätigkeit der Dichtkunst:

Dein Klagen ist gerecht, wer kann dich widerlegen?

Doch sey auch wiederum zu heilen dich bemüht;

Was wilst du deinen Schmertz noch länger nähren, hegen?

Der als ein Mörder dich zuletzt gar nieder zieht.

Wer kann des Schicksals Schluß durch Klag- und Thränen zwingen?

Viemehr verdoppelt dir der Kumer deine Pein,

Auf! und erhole dich ein männlich Lied zu singen,

Diß wird die Panace vor deine Schwermuth seyn. ${ }^{55}$

Verstummen wird hier - wie bei der Kuntsch - als ,weibliche' Haltung apostrophiert, die aktive Abkehr vom Leid durch das erneute Ergreifen des Wortes dagegen als „männlich“ (Ziegler 1728: 84).

Die Bresslerin, offenbar ebenso unwillig, die ferne Freundin zu enttäuschen, wie auch, sich nun doch noch zum Dichten aufzuraffen, reagiert in ihrem ebenfalls im Ziegler-Band abgedruckten Antwortgedicht mit einem klugen Einfall, indem sie auf ein damals beliebtes Gesellschaftsspiel zurückgreift, so genannte „bouts rimés“, ${ }^{56}$ und es solcherart abwandelt, dass sie die von der Zieglerin in ihrem Briefgedicht verwendeten Reimwörter beibehält und so ein Antwortgedicht schreiben kann, ohne selbst reimen zu müssen:

Wer kann wohl den Orcan am Firmament bezwingen?

Fürwahr des Mannes Tod verursacht meine Pein,

Auch darf mein Trauer-Thon nicht Freuden-Lieder singen,

Und keine Panace wird künfftig vor mich seyn.

Darum kann ich sonder Zug ans Reimen nicht gedencken. ${ }^{57}$

54 Christiana Mariana von Ziegler, Versuch, S. 83.

55 Ebd., S. 83-84.

56 Auch im Salon der Zieglerin war das aus den französischen Salons der so verspotteten ,précieuses` übernommene „bouts rimés“-Spiel beliebt, wie der Bericht des Leipziger Professors Philippi angibt (vgl. Katherine R. Goodman, Amazons and Apprentices. Women and the German Parnassus in the Early Enlightenment, Rochester, New York, Woodbridge 1999, S. 106).

57 Bressler in Christiana Mariana von Ziegler, Versuch, S. 88-89. 
Obwohl die Trauer der Bresslerin das ,Reimen unmöglich macht, findet sie im ,bout rimécSpiel doch eine kreative Möglichkeit, gleichwohl zu dichten, indem sie sich die Reime von der Freundin leiht. So wird die Sprachlosigkeit der Trauer gleichsam in einem Akt weiblicher Co-Autorschaft überwunden.

Als dann die Bresslerin kurz darauf in Breslau verstirbt, steht wiederum die Zieglerin - dieses mal ohne Beistand - vor dem Dilemma, sprachlose Trauer in ein Klagegedicht umzumünzen, wenn sie als Dichterin nicht doch noch verstummen will. Sie beginnt mit der topischen Beschimpfung der Musen dafür, dass diese ihr ,heute' (in der Gegenwart, die durch den ,Sprechakt' des Gedichttextes poetisch konfiguriert wird) ein "Grab-Lied“ auf ihre geliebte Gefährtin zu „singen“ auftragen, ${ }^{58}$ zitiert im Mittelteil den Unsagbarkeitstopos für das unaussprechliche Maß ihrer Trauer $^{59}$ und endet mit der ebenso topischen Versicherung des Nachruhms als Trostargument:

\section{Auf das Absterben Der Fr[au] von Breßler}

Verhaßte Tichter-Kunst! so hold ich dir gewesen,

So gram und spinne-feind wird ich dir künfftig hin,

Du kanst den Unmuth leicht aus Aug und Minen lesen,

Nicht frage nach den Quell und Ursprung meiner Schmertzen;

Küßt ich deswegen dich, daß ich nun mit Verdruß,

Derjenigen, die mir so tieff in meinen Hertzen,

Als in dem Sinne, saß, ein Grab-Lied singen muß?

$[\ldots]$

Doch da dein Fall mich heißt ein Toden-Opffer bringen,

So fällt die Feder mir vor Wehmuth aus der Hand,

Die heischre Muse weiß vor Thränen nichts zu singen,

Als diß: Mir wird durch dich was treffliches entwandt.

$[\ldots]^{60}$

Der Verlust der Schreibfeder führt hier jedoch nicht, wie bei Kuntsch, zu einem Abbruch des Gedichttextes, vielmehr wechselt die Autorin, da sie nun vor Trauer nicht mehr schreiben kann, die Modalität und singt nun ein „Klage-Lied“ (ebd., S. 24), für das sie sich - in Ermangelung der toten Freundin - nun poetischen Beistand beim personifizierten Heimatland der ,schlesischen Melpomene' holt:

Doch hörst du nicht allein mich deine Flucht beklagen,

Es singt gantz Schlesien mit mir ein Klage-Lied;

Dein Breslau kann nunmehr am allerbesten sagen,

Was durch die Breßlerin das Schicksal ihm entzieht.

$[\ldots]$

Mnemosyne läst nicht dein Gedencken sterben,

Und Pallas hängt von dir den Kiel im Tempel auf. ${ }^{61}$

58 Christiana Mariana von Ziegler, Versuch, S. 22.

59 „Ach Freundin, der Verlust steht gar nicht auszusprechen; / Der Raub ist allzu groß, der mich anjetzt betrifft" (ebd., S. 24)

60 Ebd., S. 22.

61 Ebd., S. 24-25. 
Versagt auch die Feder im Angesicht der Trauer, so klingt doch das Lied fort, und Mnemosyne, die Muse der Erinnerung, sowie Pallas Athene, die streitbare Göttin der Weisheit, bewahren - zumindest in der Binnenfiktion des gedruckt vorliegenden Gedichttextes - für immer den Nachruhm der heute fast vergessenen Breslauer Dichterin und ihrer auch nur noch wenigen bekannten Co-Autorin aus Leipzig.

\section{Literatur}

Adam, Wolfgang, Poetische und Kritische Wälder. Untersuchungen zu Geschichte und Formen des Schreibens ,bei Gelegenheit', Heidelberg 1988.

Amthor, C[hristoph], H[einrich], Poetischer Versuch Einiger Teutscher Gedichte und Übersetzungen: So wie er sie, theils in frembdem Nahmen, theils vor sich selber entworffen. Nebst einem Vor-Bericht, Worin zugleich die wieder seine Gedichte, und andere Staats-Schriffen, von einigen Ungenannten bisher ausgegossene Schmähungen bescheidentlich abgelehnet werden, Flensburg 1717.

Anz, Thomas, Tod, Angst und Trauer in der Lyrik zwischen Barock und Aufklärung, in: „Der Deutschunterricht" 54 (2002), H. 1, S. 25-35.

Becker-Cantarino, Barbara, Der lange Weg zur Mündigkeit. Frau und Literatur (1500-1800), Stuttgart 1987.

Benthien, Claudia, Barockes Schweigen. Rhetorik und Performativität des Sprachlosen im 17. Jahrhundert, München 2006.

Bodmer, Johann, Jacob, Critische Betrachtungen über die Poetischen Gemählde der Dichter, Zürich 1741.

Brandes, Helga, Lemma „Frau“, in: Werner Schneiders (Hrsg.), Lexikon der Aufklärung. Deutschland und Europa, München 1995, S. 126-128.

Brinker-Gabler, Gisela, Deutsche Dichterinnen vom 16. Jahrhundert bis zur Gegenwart. Gedichte und Lebensläufe [1978], Frankfurt a.M. ${ }^{2} 1979$.

Brockes, Barthold, Heinrich, Irdisches Vergnügen in Gott. Erster und zweiter Teil. Hrsg. v. Jürgen Rathje, Göttingen 2013.

Bunke, Simon, Mihaylova, Katerina (Hrsg.), Aufrichtigkeitseffekte. Signale, soziale Interaktionen und Medien im Zeitalter der Aufklärung, Freiburg i.Br. 2016.

Campe, Joachim, Heinrich, Wörterbuch der Deutschen Sprache, Vierter Theil: S und T, Braunschweig 1810.

Carrdus, Anna (Hrsg.), Das ,weiblich Werck' in der Residenzstadt Altenburg (1672-1720). Gedichte und Briefe von Margaretha Susanna von Kuntsch und Frauen aus ihrem Umkreis, Hildesheim, Zürich, New York 2004.

Czarnecka, Mirosława, Marianne von Bressler (1690-1728) - eine unbekannte Dichterin aus Breslau, in: Klaus Garber (Hrsg.), Stadt und Literatur im deutschen Sprachraum der Frühen Neuzeit, Bd. II, Tübingen 1987, S. 961-972.

Detken, Anke, Gekrönte Poetinnen. Gelegenheitsdichtung von Ziegler und Zäunemann, in: Sylvia Heudecker, Dirk Niefanger, Jörg Wesche (Hrsg.), Kulturelle Orientierung um 1700. Traditionen, Programme, konzeptionelle Vielfalt. Tübingen 2004, S. 263-281.

Ecker, Gisela, Trauer zeigen. Inszenierungen und die Sorge um den Anderen, in: dies. (Hrsg.), Trauer tragen - Trauer zeigen. Inszenierungen der Geschlechter, München 1999, S. 9-25. 
Goodman, Katherine R., Amazons and Apprentices. Women and the German Parnassus in the Early Enlightenment, Rochester, New York, Woodbridge 1999.

Gottsched, Johann, Christoph, Versuch einer Critischen Dichtkunst. Erster Allgemeiner Theil, Joachim Birke, Brigitte Birke (Hrsg.), Berlin, New York 1973.

Hentschel, Georg, Totenklage [Artikel], in: Manfred Görg, Bernhard Lang (Hrsg.), Neues Bibellexikon, Düsseldorf 2001, S. 908-910.

Klopstock, Friedrich Gottlieb, Ausgewählte Werke, hrsg. v. Karl August Schleiden, München 1962.

Köhler, Cornelia, Caroline, Frauengelehrsamkeit im Leipzig der Frühaufklärung. Möglichkeiten und Grenzen am Fallbeispiel des Schmähschriftenprozesses im Zusammenhang mit der Dichterkrönung Christiana Mariana von Zieglers, Leipzig 2007.

Konersmann, Ralf, Der Schleier des Timanthes. Perspektiven der historischen Semantik, Frankfurt a.M. 1994.

Martus, Steffen, Aufklärung. Das deutsche 18. Jahrhundert - ein Epochenbild [2015], Berlin ${ }^{2} 2015$.

Meid, Volker, Die deutsche Literatur im Zeitalter des Barock. Vom Späthumanismus zur Frühaufklärung (1570-1740), München 2009.

Meier, Georg, Friedrich, Beurtheilung der Gottschedischen Dichtkunst, Halle 1747.

Merkel, Kerstin, Wunder Heide, Einleitung, in: dies. (Hrsg.), Deutsche Frauen der Frühen Neuzeit. Dichterinnen, Malerinnen, Mäzeninnen, Darmstadt 2000, S. 7-17.

Müller, Monika, Schnegg, Matthias, Unwiederbringlich. Von der Krise und dem Sinn der Trauer, Göttingen 2016.

Pailer, Gaby, Poetin und Gegenstand der Poesie. Adressantenmarkierung bei Margaretha Susanna von Kuntsch (1654-1717), in: Claudia Hillebrandt, Sonja Klimek, Ralph Müller, Rüdiger Zymner (Hrsg.), Grundfragen der Lyrikologie, Bd. 1: Lyrisches Ich, Textsubjekt, Sprecher? Berlin, Boston [voraussichtlich 2019, im Druck].

Plotke, Seraina, Ziem, Alexander, Sprache der Trauer im interdisziplinären Kontext. Einführende Bemerkungen, in: dies. (Hrsg.), Sprache der Trauer. Verbalisierungen einer Emotion in historischer Perspektive, Heidelberg 2014, S. 1-17.

Quintus, Horatius, Flaccus, Ars Poetica. Die Dichtkunst. Lateinisch / Deutsch, Übers. u. m. einem Nachwort hrsg. v. Eckart Schäfer, Stuttgart 1972/2011.

Reber, Elisabeth, Gefühle nach Maß. Untersuchungen zur Trauerartikulation in den neulateinischen Epicedien für Johannes Buxtorf den Jüngeren (1599-1664), in: Seraina Plotke, Alexander Ziem (Hrsg.), Sprache der Trauer. Verbalisierungen einer Emotion in historischer Perspektive, Heidelberg 2014, S. 207-244.

Schäfer, Eckart, Anmerkungen, in: Quintus Horatius Flaccus, Ars Poetica. Die Dichtkunst. Lateinisch / Deutsch. Übers. u. m. einem Nachwort hrsg. v. Eckart Schäfer, Stuttgart 1972/2011, S. 37-54.

Segebrecht, Wulf, Das Gelegenheitsgedicht. Ein Beitrag zur Geschichte und Poetik der deutschen Lyrik, Stuttgart 1977.

Stenzel, Jürgen, ,Si vis me flere... - ,Musa iocosa mea'. Zwei poetologische Argumente in der deutschen Diskussion des 17. und 18. Jahrhunderts, in: „Deutsche Vierteljahresschrift“ 48 (1974), S. 650-671.

Stollberg-Rilinger, Barbara, Die Aufklärung. Europa im 18. Jahrhundert, Stuttgart 2000/2011.

Thomasius, Christian, Allerhand bißher publicirte Kleine Teutsche Schriften / Mit Fleiß colligiret und zusammen getragen; Nebst etlichen Beylagen und einer Vorrede, Halle 1701.

Till, Dietmar, Transformationen der Rhetorik. Untersuchungen zum Wandel der Rhetoriktheorie im 17. und 18. Jahrhundert, Tübingen 2004. 
Treyz, Sebastian, „Schämt euch der Wehmuth nicht, die feucht im Auge schimmert..." Tränen- und Trauerdispositive im Theater der Empfindsamkeit, in: Seraina Plotke, Alexander Ziem (Hrsg.), Sprache der Trauer. Verbalisierungen einer Emotion in historischer Perspektive, Heidelberg 2014, S. 245-281.

Warburg, Aby, Dürer und die italienische Antike, in: Die Erneuerung der heidnischen Antike. Kulturwissenschaftliche Beiträge zur Geschichte der europäischen Renaissance, Nachdruck der Ausgabe von 1932, hrsg. v. Horst Bredekamp, Michael Diers, Berlin 1998.

Zarnegin, Kathy, Einleitung, in: dies. (Hrsg.), buchstäblich traurig, Basel 2004, S. 7-15.

Zedler, Johann, Heinrich, Grosses vollständiges Universal-Lexicon Aller Wissenschaften und Künste $[. .$.$] , Bd. 45, 1745, 2., vollständiger photomechanischer Nachdruck durch die Akademische$ Druck- u. Verlagsanstalt, Graz 1997.

von Ziegler, Christiana, Mariana, Versuch in gebundener Schreib-Art, Leipzig 1728. 